\title{
Research on the Construction of Special Database and Data Mining in Huangdi's Internal Classic of Medicine
}

\author{
Yanda She* \\ School of Computer \\ Tonghua Normal University \\ Tonghua, China \\ E-mail:*dbcy9999@163.com
}

\begin{abstract}
This thesis, through the establishment of the special database of Huangdi's Internal Classic of Medicine, the contents of the original text of Huangdi's Internal Classic of Medicine, systematically collected and arranged the annotations of the annotator, and discussed the related data mining on the basis of the establishment of the database. The application of data mining method to the study of Huangdi's Internal Classic of Medicine can find valuable laws and knowledge, provide more scientific methods and means for the inheritance and research of traditional Chinese medicine, and expand the research content of data mining, thus laying a foundation for the standardization of traditional Chinese medicine.
\end{abstract}

Keywords-Huangdi's Internal Classic of Medicine; Database; Conceptual Model; data Mining

\section{INTRODUCTION}

Classical Chinese medicine is the foundation of the field of traditional Chinese medicine, which records a large number of valuable theoretical contents of traditional Chinese medicine. The classic books of traditional Chinese medicine, represented by the Huangdi's Internal Classic of Medicine, are the representatives of the classics of traditional Chinese medicine. However, due to its creation of a long time, the spread of the process of annotation and understanding of the classical content of a variety of different dynasties, the various medical scholars have different opinions, resulting in the rise of various theories in the field of Chinese medicine, but also for later scholars caused some confusion. In order to solve this problem, this paper establishes the special database of Huangdi's Internal Classic of Medicine, and on this basis carries on the knowledge discovery research, provides the convenient, the high efficiency, the intelligent method and the method for the scholar to refer to the ancient Chinese medicine doctorundefineds interpretation to the traditional Chinese medicine classics. In order to promote the standardization of TCM, to provide methodological support for the heritage of TCM classics.

\section{THE CONSTRUCTION OF THEMATIC DATABASE OF HUANGDI's INTERNAL CLASSIC OF MEDICINE}

In the information society, it is a prerequisite for scientific research and decision management to manage and utilize all kinds of information resources effectively [1]. More and more attention has been paid to the development and application of information resources. Database technology, as an important means and tool for managing information resources, has strong storage ability, data processing and analysis ability. For many disciplines of data processing, information retrieval, artificial intelligence and other aspects of a powerful power [2]. Database technology is the core part of all kinds of information systems, such as management information system, office automation system, decision support system, etc. It is an important technical means for scientific research and decision management. Data structure is divided into logical structure of data and physical structure of data. The logical structure is divided into hierarchical structure model, network structure model and relational structure model. The relational structure model is the main structure model that we use now. Based on the contents of Huangdi's Internal Classic of Medicine and the annotation of Huangdi's Internal Classic of Medicine, a special database of Huangdi's Internal Classic of Medicine is designed. The design process is as follows:

\section{A. Database structure design}

According to the contents of Huangdi's Internal Classic of Medicine and the characteristics of the annotators, this database adopts the relational model database, that is, the two dimensional table data is used to express the relationship between the data. This model can not only reflect the one-to-one relation between attributes, but also reflect the one-to-many relationship between attributes and the many-to-many relationship between attributes. The conceptual structure of the database is designed in the form of a conceptual structure diagram as follows:

According to the above concept structure diagram [3], we can get the logical structure of the database: the original text 
of Huangdi's Internal Classic of Medicine (serial number, volume, content, subject word), according to the concept structure and logical structure, taking SQL-Server as the database management system, Can establish each database table in the database. According to the requirements of database paradigm, these three tables have reached $3 \mathrm{NF}$ paradigm, which can reduce data redundancy and eliminate unnecessary connections [4]. Meet the database design requirements.

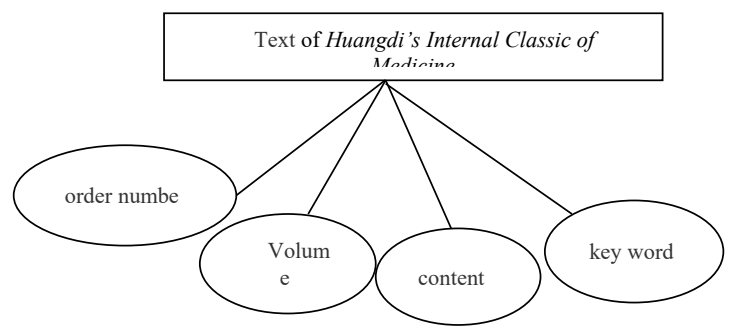

Fig. 1. A conceptual structure diagram of the original database

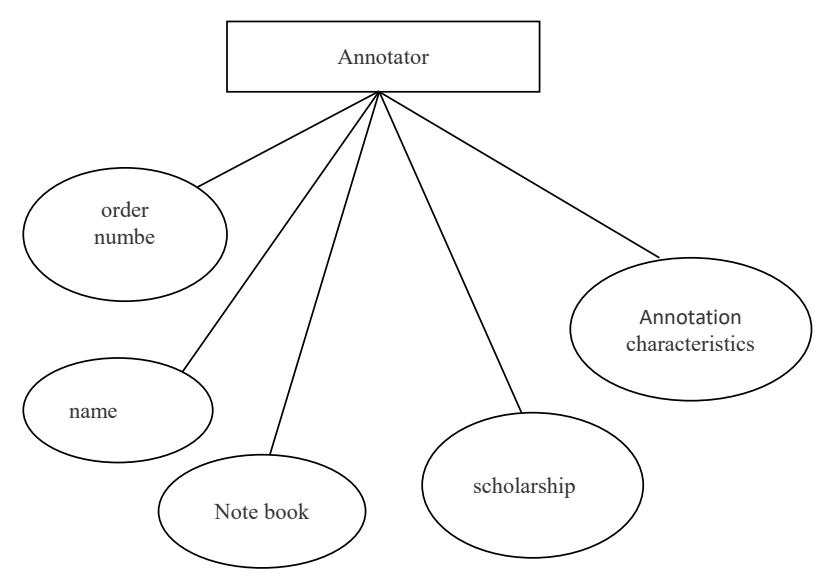

Fig. 2. Conceptual structure Chart of Huangdi's Internal Classic of Medicine annotator data Database

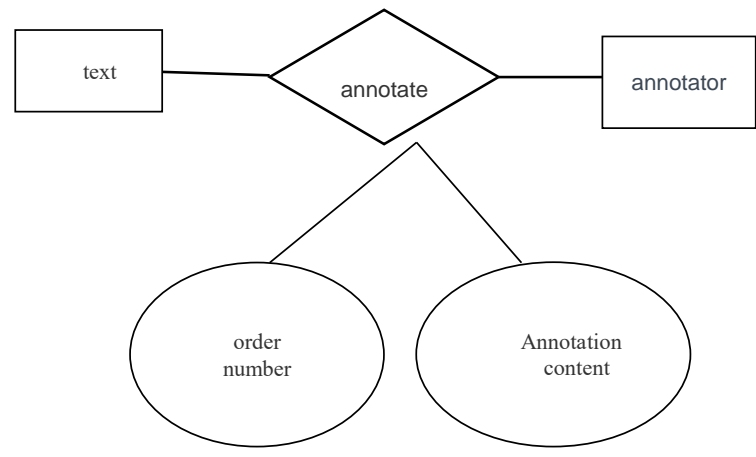

Fig. 3. Huangdi's Internal Classic of Medicine annotated database conceptual structure diagram

\section{B. System function modules}

Using Microsoft Visual C\#Microsoft Visual C \# is an object oriented programming language developed by Microsoft. It is a new programming language customized by Microsoft for. Net platform. C\#has become the mainstream program development language of. Net platform. It is designed to develop applications running on the. NETFramework. C\# programming language has the advantages of powerful syntax, object oriented, simple structure, type safety and so on. While maintaining the same syntax form of $\mathrm{C}$ language, the rapid development of application program can be realized.

The SQL Servers of this database system is used to manage the database, and Microsoft Visual C \# is used to develop the Windows window application program which is easy to operate, which makes the complex database transparent and ensures the integrity of the data. Usability and recoverability, and easy to operate, the database can be manipulated by the application program, and scientific inquiry and statistical analysis can be carried out on the theory of liver storage and annotated notes of the Inner Classic. To provide reliable technical support for further discussion of relevant theories.

The main contents are as follows:

(1) the data input and maintenance module realizes the management of user account, which is divided into administrator and ordinary user. It can input and store the annotation of liver storage image theory and 14 notes, and can be added, deleted and modified by data maintenance function.

(2) the query module uses Microsoft Visual C \# technology to set up the Huangdi's Internal Classic of Medicine note this query system, the system can provide the convenient and quick query function, can from the theme word, Subtheme words and other multi-angle single and multi-item combination retrieval to realize the classification of query results browsing.

(3) the data mining module collects and analyzes the results of the database query system, realizes the vertical and 
horizontal analysis of all the liver image theory and the annotatorsundefined notes in Huangdi's Internal Classic of Medicine [5], so as to find the unknown laws and knowledge to be stored in the knowledge base.

\section{DATA MINING RESEARCH}

\section{A. Data mining}

Data mining is an interdisciplinary subject, which is widely used with the development of computer technology. The research of data mining begins with the concept of finding useful patterns from databases, which refers to the nontrivial process of identifying valid, novel, potentially useful and ultimately understandable patterns in the database. The task of data mining is to find and extract hidden, unknown, potentially useful and understandable rules and patterns from a large number of data. Therefore, data mining is widely used to solve data classification and data clustering [6]. Decline and prediction, correlation and correlation, sequence discovery, description and discrimination, time series analysis, etc.

In recent years, data mining is also widely used in the field of traditional Chinese medicine [7]. For example, the data of prescription and traditional Chinese medicine are standardized, database is built, and the law of compatibility of prescription and Chinese medicine is analyzed, which provides reference for clinical practice [8].

\section{B. Application of data mining in Huangdi's Internal Classic of Medicine}

Based on the establishment of the special database of Huangdi's Internal Classic of Medicine, the method of data mining is applied to the study of Huangdi's Internal Classic of Medicine, which can mine the hidden and potential knowledge in the original text of Huangdi's Internal Classic of Medicine and the relevant annotations. We can use the arithmetic of word frequency statistics to objectively analyze the frequency of terms appearing in Huangdi's Internal Classic of Medicine and the correlation between the terms, so as to provide a new method for the study of Huangdi's Internal Classic of Medicine. It provides a scientific reference for intelligently extracting knowledge ofHuangdi's Internal Classic of Medicine.

In this paper, fourteen annotators and 19 annotated books are selected from the annotators who have annotated Huangdi's Internal Classic of Medicine in the past dynasties. The chronological order is as follows: Yang Shangshan (Tang): Huangdi's Internal Classic of Medicine Tai Su; Wang Bing (Tang): The annotation of Huangdi's Internal Classic of Medicine; Hua Shou (Yuan): Du Suwen Chao; Ma Shis (Ming): Huangdi's Internal Classic of Medicine Sutra and Huangdi's Internal Classic of Medicine Lingshu note card issue; Wu Kun (Ming): The annotation of Wu to suwen of Huangdi's Internal Classic of Medicine; Zhang Jiabin (Ming): Classical Classics; Li Zhongzi (Ming): essential knowledge of the Inner Classic; Zhang Zhicong (Qing): Huangdi's Internal Classic of Medicine Sutra
Superior Sutra Note Section explanation; Gao Shizong (Qing): Huangdi's Internal Classic of Medicine Sutra Note Section explanation; Huang Yuanyu (Qing): the suspensions of the Suwen and the suspensions of the Lingshu; Danboyuanjian (Japan): the knowledge of the Suwen and the knowledge of the Lingshu; Chen Xiuyuan (Qing): the Lingsu Festival should be shallow Note.

It is impossible to study Huangdi's Internal Classic of Medicine without the help of ancient annotations, because the ancients came closer to the era of the original work, the language gap was smaller and the understanding was more accurate [9]. Therefore, it is an essential way and method to study Huangdi's Internal Classic of Medicine by referring to the annotated book, choosing good and following through. For the study of the theory of liver storage image in the Internal Classic, with the help of the notes of ancient doctors, we can clear up some fuzzy understandings of the theory of Huangdi's Internal Classic of Medicine, and thus more accurately grasp the essential connotation of the theory of Huangdi's Internal Classic of Medicine[10]. The inheritance of the Huangdi's Internal Classic of Medicine can play a role in clearing up the source.

It is based on the theoretical annotation of the original text of Huangdi's Internal Classic of Medicine to study in this paper, and introduces the data mining technology to mine the relevant annotations of Huangdi's Internal Classic of Medicine.. Potential knowledge. This paper constructs the data mining model of the special database of Huangdi's Internal Classic of Medicine. as shown in figure 4.

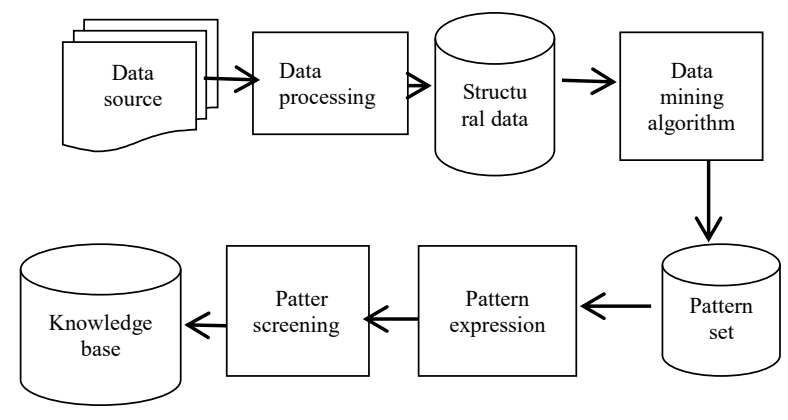

Fig. 4. Data mining model

In this paper, 17 terms related to liver storage are selected for word frequency analysis. It is found that the highest frequency is "liver-qi", and the frequency is up to 52 times. When discussing the physiology and pathology of liver, there is much discussion from the aspect of Qi, which is related to the philosophy of that time. But "liver-fire" frequency is lowest, only once. It is concluded that there is no "liver-fire" which describes the pathological phenomena in modern times in the "Inner Classic" era, and the theory of liver-fire is played by the physicians in describing the pathological manifestations of the liver. 
This paper analyzes the characteristics of annotator annotated Huangdi's Internal Classic of Medicine through the special database of Huangdi's Internal Classic of Medicine as follows:

The features of Yang Shangshan's annotation to Huangdi's Internal Classic of Medicine is characterized by strong Buddhist color and reflects the thought of Taoism. featuresof Wang Bing's annotation to Huangdi's Internal Classic of Medicine is the ancient literature is taken as the documentary evidence of its annotation, so that the contents of its annotation become clearer and clearer, and its annotation has a strong Taoist thought; Annotate the theory of Huangdi's Internal Classic of Medicine with the theory of Wuyunliuqi. The features of Hua Shou's annotation to Huangdi's Internal Classic of Medicine can be summed up as follows: deep clinical foundation, emphasis on combining theory with practice; emphasis on annotation from meridian theory; classification of Hua Shou into categories, and the classification method of copy reading plays a leading role for doctors of later generations. The characteristics of $\mathrm{Ma}$ Shi's annotation to Huangdi's Internal Classic of Medicine Internal can be summarized as follows: Suwen and Lingshu are mutually annotated, and the annotated text is detailed and thorough. The characteristics of Wu Kun's annotation to Huangdi's Internal Classic of Medicine: expounding new ideas, not adding the former notes, and closely combining the notes with clinical practice. The characteristics of Zhang Jiabin's annotation to Huangdi's Internal Classic of Medicine: combined with the theory of luck to elucidate the pathogenesis; emphasize the origin of essence and blood, liver and kidney, and pay attention to the pathogenic factors of emotion. The characteristics of Li Zhongzi's annotation to Huangdi's Internal Classic of Medicine: pay attention to the nature of the Tibetan image; the commentary is concise and concise. The characteristics of Wang Ang's annotation of Huangdi's Internal Classic of Medicine can be summed up as follows: the Suwen is the main part, the Lingsu interacts with each other, and the theory of Wuyunliuqi is attached importance to. The characteristics of Zhang Zhicong's annotation of Huangdi's Internal Classic of Medicine can be summed up as follows: brainstorming, choosing good from the best; citing the Sutraundefineds strongholds, indicating the source. The features of Gao Shizong's annotation of Huangdi's Internal Classic of Medicine can be summed up as follows: plain text, simple and clear comments, unique views, dare to break through. The features of Yao Zhian's annotation of Huangdi's Internal Classic of Medicine can be summed up as follows: characteristics: choose good and follow, cut to the Sutra. The features of Huang Yuanyu's annotation of Huangdi's Internal Classic of Medicine can be summed up as follows: advocate the ascending and descending flow of gas machine, elaborate the pathology in detail and clear. The features of Danbo YuanJian's annotation of Huangdi's Internal Classic of Medicine can be summed up as follows: general evaluation of the various, choose good and follow, integration. The features of Chen Xiuyuan's annotation of
Huangdi's Internal Classic of Medicine can be summed up as follows: annotation is easy to understand, return to Bo is about.

\section{CONCLUSION}

This paper systematically collects and arranges the contents of the original text of Huangdi's Internal Classic of Medicine and the annotations of the annotators through the establishment of the special database of Huangdi's Internal Classic of Medicine. Based on the traditional Chinese medicine research and the development of a data mining system, it can find valuable knowledge and provide more scientific research methods and means for the field of traditional Chinese medicine. At the same time, the research content of data mining system is expanded, so as to lay a foundation for standardization and standardization of traditional Chinese medicine.

The theory of Huangdi's Internal Classic of Medicine and the annotatorundefineds annotation are constructed into the structured form of database, one is that it is convenient to manage the digital resources of TCM, the other is the convenience of knowledge discovery. The method of combining quantitative analysis and qualitative analysis in the process of knowledge discovery is not only to believe in the "mining" of information from the database, but to find useful knowledge through human discrimination. The purpose of knowledge discovery is to improve efficiency, but not to replace the main role of human.

\section{REFERENCES}

[1] Qian Xuezhong, Principle and application of database . Beijing: Beijing University of posts and Telecommunications Press, 2007.

[2] Bain T, SQL server 2000 data Warehouse and Analysis Services. Beijing: China Electric Power Press: 2003.

[3] Wang Shan, Database Technology and online Analytical processing. Beijing: Beijing Science Press, 2017.

[4] Wang Shan, sa Shixuan, introduction to Database system Beijing: higher Education Press, 2014.

[5] Wang Bing. Notes on the essence of Huangdiundefineds Internal Classic. Beijing: Xueyuan press. 2004.

[6] Ni Zhiwei, Cai Qingsheng. Neural network expert system based on knowledge discovery technology. Journal of Xiamen University (Natural Science Edition), 2000N.

[7] Chen Liping, Li Jian-sheng, Cai Yongmin, et al. Train of thought and method of constructing and excavating TCM pulmonary disease database. Chinese Journal of traditional Chinese Medicine, 2017 (12): 5530-5534

[8] Liu Jia-hui, Wei Zhi-hui, Lu Dong-yong, et al. Study on the Drug use of Old Chinese Medicine for Primary Hepatocellular carcinoma based on data Mining. Chinese Journal of traditional Chinese Medicine, 20161 (1) $58-61$

[9] Xiong Jibo. See Note on Huangdi's Internal Classic of Medicine. China Journal of Chinese Medicine, 2014-04-21 (004).

[10] Hu Ling, Jiao Zhenlian. A brief account of the Origin of the Huangdi's Internal Classic of Medicine, Shanxi traditional Medicine, 2013 (05): 616-617. 